\title{
Gesellschaft für Phytotherapie e. V. veranstaltete erfolgreich das 4. Modul der Fortbildungsreihe für Ärzte und Apotheker
}

Nachdem im Frühjahr 2016 das 3. Modul „Phytopharmaka und Phytotherapie“ erfolgreich durchgeführt wurde, fand vom 23.-25. September 2016 das 4. Modul dieser Fortbildungsreihe wiederum im Lindner Sport \& Aktiv Hotel Kranichhöhe, Much/Bergisches Land statt. Mit diesem Modul wurde die erste Fortbildungsreihe der Gesellschaft für Phytotherapie e. V. erfolgreich beendet.

Die wissenschaftliche Leitung übernahm Frau Prof. Karen Nieber (Vorsitzende des wiss. Kuratoriums der GPT), die ärztliche Leitung lag in den Händen von Frau Prof. Karin Kraft (Präsidentin der GPT). Für die Organisation war wieder Frau Cornelia Schwöppe (Schatzmeisterin der GPT) verantwortlich. Den Schwerpunkt des 4. Moduls bildeten Vorträge zur Phytotherapie besonderer Personengruppen. 20 Ärzte und Apotheker aus Deutschland und Österreich hatten sich zu diesen interessanten Themen angemeldet.

Nach dem Einführungsvortrag „Phytopharmaka in Leitlinien“ stand am ersten Tag die Phytotherapie bei Kindern auf dem Programm. Dr. Andreas Franken stellte zuerst die „Initiative Arzneimittel für Kinder (IKAM)“ vor, dessen Vorsitzender er ist. Die IKAM wurde vom Bundesverband der Arzneimittel-Hersteller (BAH) ins Leben gerufen und ist als gemeinnütziger Verein eingetragen. Herr Dr. Franken diskutierte mit den Teilnehmern über Phytopharmaka, die besonders für Kinder geeignet sind und solche, die nicht angewendet werden sollten. Auch Dosierungsprobleme, die Frage, warum es so wenige zugelassene Arzneimittel für Kinder gibt und neue Studien zur Anwendung von Phytopharmaka bei ADHS wurden diskutiert.

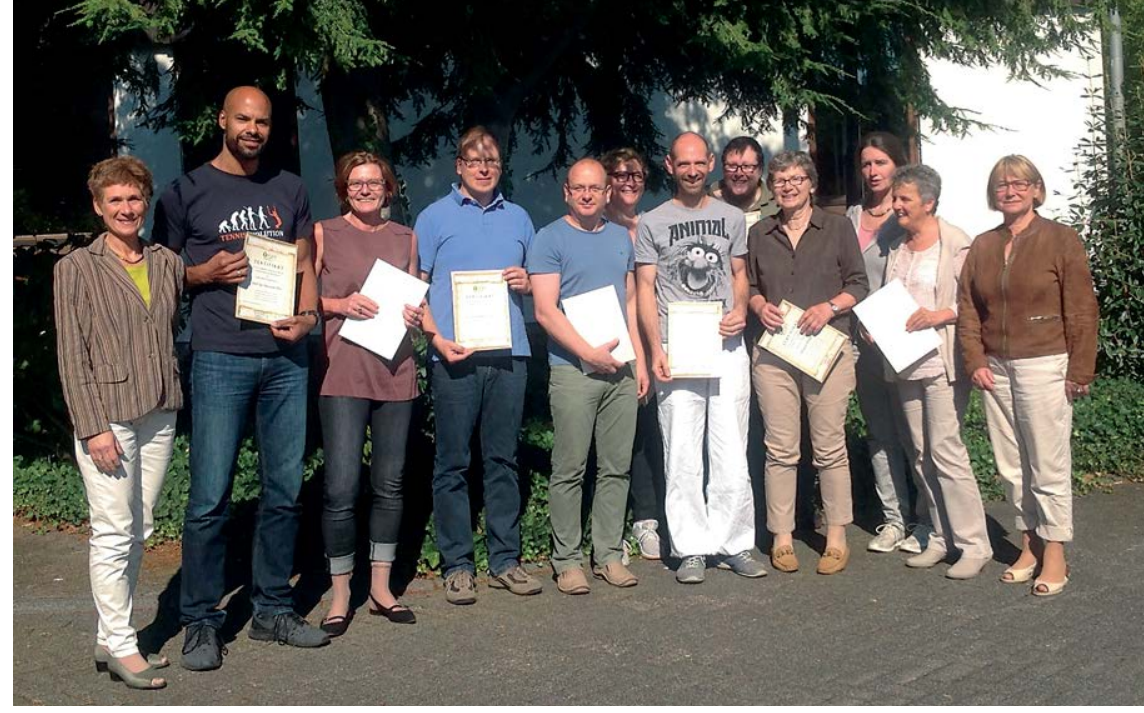

Teilnehmer, die das Zertifikat der GPT nach erfolgreichem Abschluss der 4 Module erhalten haben. (c) Nieber

\section{PhytoVIS}

Der Nachmittag wurde mit der Vorstellung des Projektes „PhytoVIS - Versorgungsforschung mit Phytopharmaka“ durch Frau Cornelia Schwöppe fortgesetzt. Die Kooperation Phytopharmaka hat in Zusammenarbeit mit dem Institut IMSIE (Köln) 2012 die Datenbank „PhytoVIS“ ins Leben gerufen, um das Erfassen von Daten in produkt- und indikationsübergreifender Weise zu ermöglichen. Das Ziel ist es, Daten über den Nutzen der Behandlung mit Phytopharmaka bei Patienten unterschiedlicher Altersgruppen und Krankheitsbilder zu sammeln (Versorgungsforschung). „PhytoVIS“ steht für Vertrauen, Information und Sicherheit bei der Anwendung pflanzlicher Arzneimittel und ist eine retrospektive Online-Befragung zur Untersuchung der Anwendungserfahrungen mit pflanzlichen Arzneimitteln. PhytoVIS ist eine Plattform für registrierte epidemiologische
Studien mit ENCePP-Siegel. Die Geschäftsführerin der Kooperation Phytopharmaka berichtete, dass bisher knapp 20000 Eintragungen vorliegen und dass die Auswertung demnächst beginnt.

\section{Bedarf: Studien zur Phyto-Dermatologie}

Der zweite Tag begann mit dem Thema „Besondere Aspekte bei der Anwendung von Phytos bei Jugendlichen“. Ausgehend von einer Analyse der Situation in Deutschland konzentrierte sich Frau Prof. Nieber auf neurologische Erkrankungen (Depression/Angststörungen, Schlaf-, Essstörungen) und Hauterkrankungen. Die Referentin machte deutlich, dass trotz jahrhundertelangen Erfahrungen mit dem Einsatz von pflanzlichen Heilmitteln bislang nur wenige eine Empfehlung bei dermatologischen Problemen erhalten ha- 


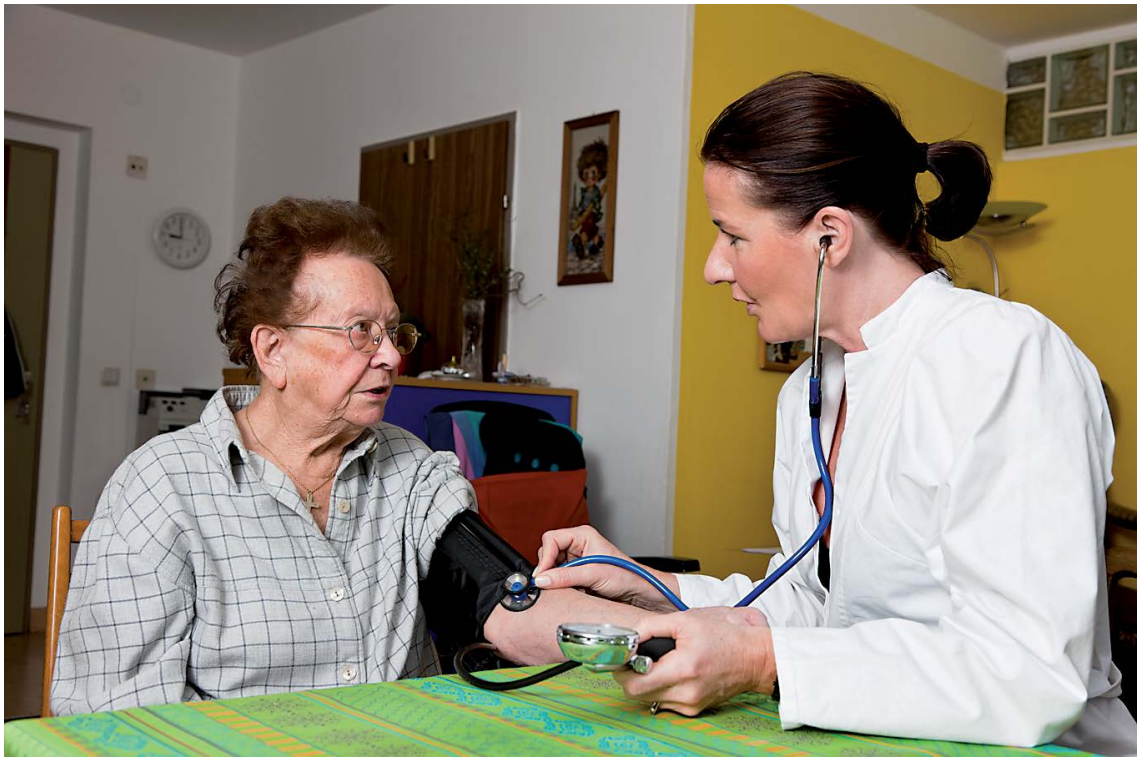

Senioren im Fokus der Phytotherapie: Sie leiden häufig unter potenziell inadäquaten Medikamenten in Verbindung mit Polypharmazie. (c) Erwin Wodicka - BilderBox.com

ben, da die wissenschaftliche Studienlage gering ist und gute klinische Studien fehlen. Nach derzeitigem Erkenntnisstand können zur Behandlung Phytopharmaka nur bei leichten Formen eingesetzt werden. Bei schweren Verlaufsformen von Hauterkrankungen gibt es bisher weder überzeugende Studien noch entsprechende Erfahrungswerte.

\section{Erfahrungsaustausch: Phytotherapie in der Schwangerschaft}

Der Vormittag wurde mit zwei Vorträgen zur Anwendung von Phytopharmaka bei Schwangeren und Stillenden fortgesetzt. In beiden Vorträgen wurden wesentliche Probleme während der Schwangerschaft wie Schlafstörungen, Meteorismus, Obstipation, Sodbrennen oder Emesis gravidarum sowie Probleme beim Stillen (z. B. die Anwendung von Stillöl) intensiv diskutiert. Auch die Anwendung von Phytopharmaka bei der Dammschnittpflege war Gegenstand eines Vortrages. Abschließend wurde eine Tabelle präsentiert, in der Pflanzen aufgeführt sind, die in irgendeiner Weise fruchtschädigende oder auf den Fötus ungünstige Wirkungen zeigen können und deshalb vermie- den werden sollten. In der nachfolgenden Diskussionsrunde wurden Erfahrungen aus der täglichen Praxis der Teilnehmer zum Thema „Wie beurteilen Sie die Anwendung von Phytopharmaka in der Schwangerschaft" ausgetauscht. Dieser sehr intensive Erfahrungsaustausch zwischen Ärzten und Apothekern erbrachte für alle Teilnehmer einen großen praxisrelevanten Nutzen und wurde sehr positiv bewertet.

Der Nachmittag war dem Thema „Phytotherapie bei Senioren“ gewidmet. Durch altersbedingte Veränderungen in der Pharmakodynamik und Pharmakokinetik sowie durch die Polypharmazie stellt diese Altersgruppe eine besondere Herausforderung für den Arzt und Apotheker dar. Es besteht ein erhöhtes Risiko von Interaktionen und unerwünschten Arzneimittelwirkungen. Dies gilt nicht nur für die Verordnung mehrerer Arzneimittel, sondern auch für die alleinige Gabe bestimmter Wirkstoffe, die als potenziell inadäquate Medizin (siehe Priscus-Liste) eingestuft werden. Hier zeigen sich Phytopharmaka als echte Alternative besonders für leichte bis mittelschwere Beschwerden bei chronischen Erkrankungen, so das Resümee der Referentin.

\section{Teilnehmerwunsch}

\section{Aromatherapie}

Den Abschluss des zweiten Tages bildete ein fakultativer Vortrag, der auf Wunsch der Teilnehmer in das Programm aufgenommen wurde. Frau Marita Kunkel, Fachärztin für Innere Medizin, Geriatrie und Naturheilverfahren am Herz-Jesus-Krankenhaus Lindlar und Teilnehmerin des Fortbildungskurses, führte in die Aromatherapie ein. In einem mit großer Begeisterung vorgetragenen Referat, ergänzt durch einige „Kostproben“, wurde die Wirkung von ätherischen Ölen an einfachen Beispielen demonstriert. Ätherische Öle finden heute Verwendung in Form von Ganz- und Teilbädern, als Kompressen und Wickel, zum Inhalieren oder als Massageöle. An praktischen Beispielen demonstrierte Frau Kunkel die therapeutischen Möglichkeiten und die Kursteilnehmer konnten sich selbst von der Wirkung einiger ätherischer Öle überzeugen.

Am dritten Tag beschäftigte sich Frau Prof. Nieber in einem Vortrag mit der Frage „Alkohol in Phytopharmaka - sinnvoll oder schädlich?“. Es wurden Ergebnisse aus neuen Studien gezeigt, die eindeutig bestätigen, dass der Alkoholgehalt der meisten pflanzlichen Arzneimitteln bei korrekter Einnahme geringer ist als der Alkoholgehalt in verschiedenen Lebensmitteln, sodass eine schädigende Wirkung auch bei Kindern nicht zur erwarten ist.

Den Abschluss des 4. Moduls bildete der Vortrag „Das Arzneipflanzenlexikon Arzneipflanzen entdecken“. Herr Prof. Jürgen Reichling (Heidelberg) gab einen historischen Überblick über die Entwicklung von Arzneipflanzenlexika und stellte den Aufbau und Inhalt des Arzneipflanzenlexikons der Kooperation Phytopharmaka vor. Dieses Nachschlagewerk erlaubt einen sehr guten Überblick über Heilpflanzen und kann den Kursteilnehmern dienen, ihr Wissen zu erweitern oder in der Praxis sehr schnell Informationen über Biologie, Inhaltsstoffe, Wirkungen, mögliche Nebenwirkungen und Indikationen zu erhalten. 


\section{"Es hat sich gelohnt"}

Ein Höhepunkt des Moduls war am letzten Tag die Verleihung der Zertifikate der GPT an 10 Kursteilnehmer, die alle 4. Module erfolgreich abgeschlossen hatten. Durch Frau Prof. Nieber und Frau Dr. Braunewell (Vorstandsmitglied der GPT) wurden die Zertifikate überreicht. In den Dankesworten dieser Teilnehmer wurden besonders die sehr gute Organisation und die interessante Themenverteilung sowie die stimulierenden Diskussionen hervorgehoben. Eindeutiges Credo war, dass sich die Teilnahme gelohnt hat und dass die vielen neuen Erkenntnisse eine Bereicherung für die tägliche Arbeit sind.

\section{Neuer Zyklus 2017}

Der Vorstand der GPT hat beschlossen, die Fortbildungsreihe in gleicher Form in

Ein Dankeschön an Frau Prof. Dr. Karen Nieber

Frau Prof. Karen Nieber nahm anlässlich des erfolgreichen Abschlusses der ersten Fortbildung der GPT für Ärzten und Apotheker ein besonderes Dankeschön entgegen. Es wurde von Dr. Heidi Braunewell und Cornelia Schwöppe im Namen des Vorstands der Gesellschaft für Phytotherapie überbracht. Prof. Nieber organisierte als Vorsitzende des wissenschaftlichen Kuratoriums der GPT in vorbildlicher Weise eine praxisorientierte und wissenschaftlich hochkarätig besetzte Fortbildung. Vier modulare Wochenenden werden auch in Zukunft zum Zertifikat „Phytotherapeut/in“ führen. Informationen dazu unter http://www.phytotherapy.org/de/termine

2017 fortzusetzen. Das 1. Modul des neuen Zyklus mit den Schwerpunkten Phytopharmaka bei psychiatrischen, rheumatischen und Hautkrankheiten wird vom 31.3.-2.4.2017 stattfinden. Flyer hierzu werden in Kürze veröffentlicht.
Weitere Informationen zu dem 1. Modul 2017 finden sich demnächst auf der Homepage der GPT unter http://www. phytotherapy.org.

Prof. Dr. Karen Nieber, Cornelia Schwöppe 